\title{
Energy relaxation in disordered charge and spin density waves
}

\author{
R. Mélin ${ }^{(1) *}$, K. Biljaković( ${ }^{(2)}$ and J.C. Lasjaunias ${ }^{(1)}$ \\ (1) Centre de Recherches sur les Très Basses Températures (CRTBT) ${ }^{\dagger}$, \\ Boîte Postale 166, F-38042 Grenoble Cedex 9, France \\ (2) Institute of Physics, Hr-10 001 Zagreb, P.O. Box 304, Croatia
}

\begin{abstract}
We investigate collective effects in the strong pinning model of disordered charge and spin density waves (CDWs and SDWs) in connection with heat relaxation experiments. We discuss the classical and quantum limits that contribute to two distinct contribution to the specific heat (a $C_{v} \sim T^{-2}$ contribution and a $C_{v} \sim T^{\alpha}$ contribution respectively), with two different types of disorder (strong pinning versus substitutional impurities). From the calculation of the two level system energy splitting distribution in the classical limit we find no slow relaxation in the commensurate case and a broad spectrum of relaxation times in the incommensurate case. In the commensurate case quantum effects restore a non vanishing energy relaxation, and generate stronger disorder effects in incommensurate systems. For substitutional disorder we obtain Friedel oscillations of bound states close to the Fermi energy. With negligible interchain couplings this explains the power-law specific heat $C_{v} \sim T^{\alpha}$ observed in experiments on CDWs and SDWs combined to the power-law susceptibility $\chi(T) \sim T^{-1+\alpha}$ observed in the CDW o-TaS 3 .

PACS numbers: 71.45.Lr, 05.70.Ln, 63.50.+x, 75.30.Fv
\end{abstract}

\section{INTRODUCTION}

There exist many examples of systems showing slow relaxation and ageing: spin glasses ${ }^{1.2}$, disordered dielectrics 3.4 .5 , supercooled liquids ${ }^{6}$, etc ... Charge density waves (CDWs) and spin density waves (SDWs) ${ }^{7.8 .9 .10}$ show "interrupted ageing" 11 , meaning that there exists an upper bound $\tau_{\max }$ to the relaxation times. The protocol of ageing experiments in CDWs and SDWs is the following: the system is equilibrated at the temperature $T$ (one waits for a time longer than $\tau_{\max }$ ). At time $t=0$ the temperature is changed from $T$ to $T+\Delta T$ where $\Delta T>0$ is very small compared to $T$. The temperature is kept constant until the waiting time $t_{w}$ where it is brought back to $T$. The heat flows between the CDW or SDW sample and the cold source are recorded as a function of time. Ageing in the thermal response takes place even for very small values of $\Delta T$. Since very small temperature variations are applied in the experiment it is reasonable to suppose that the size of the correlated objects does not evolve in time and that the thermal response is due solely to the variation in the population of metastable states. This can be contrasted with the coarsening dynamics where the size of correlated domains increases with time.

In a recent work $^{12}$ we applied the idea of dynamical renormalization group $\frac{13.14}{4}$ to calculate the spectrum of relaxation times of a model of disordered CDW or SDW $15.16 .17 \cdot 18.19 .20 .21 .22 .23 .24$, including interactions among bisolitons. A drawback of this approach $\frac{12}{2}$ is that we supposed a coarsening dynamics following a quench

\footnotetext{
*E-mail: melin@grenoble.cnrs.fr

${ }^{\dagger}$ U.P.R. 5001 du CNRS, Laboratoire conventionné avec l'Université Joseph Fourier
}

from high temperature, a situation that is not realized in experiments, and we were not able to address the waiting time dependence of the relaxation time spectra. One goal of the present article is to address these issues that were left open in our previous work ${ }^{12}$, and to put on a microscopic basis the random energy-like (REM-like) trap model that was proposed in Ref. 12, and inspired from trap models developed for glasses and spin glasses 11.25 .

More specifically we show here that heat relaxation experiments can be described by assuming two types of defects (strong pinning and substitutional impurities), corresponding to the "classical" limit where the CDW or SDW is viewed as a classical elastic medium with bisolitons generated by strong pinning impurities distributed at random, and to the "quantum" limit where solitons due to substitutional disorder interact quantum mechanically by excitations of the gaped background. The existence of two effects is in agreement with the experimental observation that the low temperature out-of-equilibrium specific heat can be decomposed into three contributions: (i) the $C_{v} \sim 1 / T^{2}$ tail of a Schottky anomaly at very low temperature (typically for $T \lesssim 100 \div 300 \mathrm{mK}$; the upper bound depends on the amplitude of the $1 / T^{2}$ contribution); (ii) a $C_{v} \sim T^{\alpha}$ power-law specific heat with $\alpha \simeq$ $0.3 \div 1.2$ at intermediate temperatures $(0.1 \lesssim T \lesssim 1 \mathrm{~K})$; and (iii) the "trivial" contribution of phonons $C_{v} \sim T^{3}$ at high temperature $(T \gtrsim 1 \mathrm{~K})$. By Schottky anomaly we mean that the equilibrium specific heat of a two-level system with energies $E_{0}$ and $E_{0}+\Delta E$ is given by

$$
C_{v}(T)=\frac{(\Delta E)^{2}}{4 T^{2}} \frac{1}{\cosh ^{2}(\Delta E / 2 T)}
$$

having a maximum (called a Schottky anomaly) at $T_{\max } \simeq 0.416 \Delta E$. The specific heat is approximately equal to $C_{v} \simeq(\Delta E)^{2} / 4 T^{2}$ in the large temperature tail.

Following Ref. 23, the contribution (i) is interpreted in terms of two-level systems due to strong pinning impuri- 
ties. The contribution (ii) is interpreted as midgap states interacting through Friedel oscillations. Friedel oscillations of a single impurity were probed directly by x-ray diffraction experiments in Ref. 26. Another evidence in favor of the coexistence of strong pinning and substitutional impurities is that the CDW compound o- $\mathrm{TaS}_{3}$ can be doped by $\mathrm{Nb}$, a substitutional impurity. This changes only the amplitude of the $C_{v} \sim T^{\alpha}$ contribution, but leaves unchanged the $C_{v} \sim 1 / T^{2}$ contribution ${ }^{27}$, suggesting that the power-law contribution is related to substitutional disorder. Even though not affected by substitutional disorder we do not interpret the $C_{v} \sim 1 / T^{2}$ as a property of the pure compound. A nuclear hyperfine interaction can be excluded from the systematic study of many different CDW compounds ${ }^{7}$. We thus relate the $C_{v} \sim 1 / T^{2}$ contribution to strong pinning impurities ${ }^{23}$, even though the microscopic nature of these impurities is not well understood experimentally (see Ref. 28 for a study of ESR spectroscopy in o- $\mathrm{TaS}_{3}$ ).

The commensurate organic spin-Peierls compound $(\mathrm{TMTTF})_{2} \mathrm{PF}_{6}$ showing slow relaxation ${ }^{29}$ contrasts with the inorganic spin-Peierls compound $\mathrm{Cu}_{1-x} \mathrm{Zn}_{x} \mathrm{GeO}_{3}{ }^{30.31 .32 .33}$ showing antiferromagnetic ordering. We argue that the difference lies in the different nature of disorder. Substitutional disorder relevant to $\mathrm{Cu}_{1-x} \mathrm{Zn}_{x} \mathrm{GeO}_{3}$ is qualitatively different from strong pinning impurities in CDWs and SDWs. The spin-Peierls compound $\mathrm{Cu}_{1-x} \mathrm{Zn}_{x} \mathrm{GeO}_{3}$ has a fast dynamics, with a "microscopic" time presumably comparable to the one of spin glasses $\left(\tau_{0} \simeq 10^{-12} \mathrm{sec}\right)$ whereas in CDW and SDW compounds we have $\tau_{0} \simeq 1$ sec for the thermally activated process. This indicates that rather different mechanisms are at work, identified here are substitutional or strong pinning disorder. We generalize to the incommensurate case the model of substitutional disorder introduced in Ref. 34. In this model the solitons are due to domain walls between two degenerate ground states since the impurity site can be removed from the chain (see Ref. 34 and section III), therefore leaving randomly distributed domain walls in the chain from which the impurities sites have been removed. The specificity of this model (as opposed to the strong pinning model) is that there are no metastable states of bisolitons like in the strong pinning model. The substitutional impurities do not contribute to the slow dynamics of bisolitons in the strong pinning limit but are expected to contribute to collective pinning of the phase of the density wave. However we consider here temperatures much lower than the glass transition temperature ${ }^{35.36}$ so that interactions among solitons are the only remaining collective effects. For substitutional disorder we find interactions among solitons due to Friedel oscillations. We obtain similarly to Ref. 34 a power-law specific heat $C_{v}(T) \sim T^{\alpha}$ and a susceptibility $\chi(T) \sim T^{-1+\alpha}$ in agreement with existing experiments on the CDW o- $\mathrm{TaS}_{3}{ }^{27}$.

The article is organized as follows. In section [11 we investigate a classical model of collective effects in a dis- ordered CDW. Quantum effects are investigated in section III Final remarks are given in section IV

\section{CLASSICAL LIMIT (STRONG PINNING IMPURITIES)}

\section{A. Hamiltonian}

Let us start with a classical model of disordered $\mathrm{CDW}^{15,16,17,18,19,20,21,22,23,24}$. To derive the $1 \mathrm{D}$ projection of the Hamiltonian of the phase of the CDW in the mean field approximation we follow the recent review by Brazovskii and Nattermann ${ }^{24}$ and consider a system of coupled chains with a phase $\varphi_{n}(y)$ in chain $n$ ( $y$ is the coordinate along the chain axis).

$$
\begin{aligned}
\mathcal{H} & =\frac{\hbar v_{F}}{4 \pi} \sum_{n} \int d y\left(\frac{\partial \varphi_{n}(y)}{\partial y}\right)^{2} \\
& +\sum_{n, m} w_{m, n} \int d y\left[1-\cos \left(\varphi_{n}(y)-\varphi_{m}(y)\right)\right] \\
& -\sum_{n, i} V_{i}^{(n)}\left[1-\cos \left(Q y_{i}^{(n)}+\varphi_{n}\left(y_{i}^{(n)}\right)\right)\right]
\end{aligned}
$$

where the sum in the last term runs over all impurities, $v_{F}$ is the Fermi velocity along the chain axis, $w_{m, n}$ corresponds to the commensurate energy or interchain coupling, $V_{i}^{(n)}$ is the pinning energy of the impurity number $i$ in chain $n$, and $Q=2 k_{F}$ is the wave vector of the CDW. Assuming dilute impurities we suppose that the chain $n=0$ with $\varphi_{0}(x) \equiv \varphi(x)$ is coupled to neighboring chains with $\varphi_{m}(x)=0$. We arrive at the effective 1D Hamiltonian

$$
\begin{aligned}
\mathcal{H} & =\frac{\hbar v_{F}}{4 \pi} \int d y\left(\frac{\partial \varphi(y)}{\partial y}\right)^{2}+w \int d y[1-\cos \varphi(y)] \\
& -\sum_{i} V_{i}\left[1-\cos \left(Q y_{i}+\varphi\left(y_{i}\right)\right)\right]
\end{aligned}
$$

Metastable states due to the competition between the commensurate potential and the pinning energy were first discussed by $\mathrm{Abe}^{\underline{19}}$ in a different approach. Solitons and the transition to a 3D density wave glass were discussed by Fukuyama ${ }^{15}$, and bisolitons were discussed by Larkin 22 and Ovchinikov ${ }^{23}$.

\section{B. No impurity: $2 \pi$-solitons}

Without impurities there exist solutions minimizing the energy (5) in which the the phase winds by $\pm 2 \pi$ within a length $\xi^{15,22}$ :

$$
\tan \left(\frac{\varphi(y)}{4}\right)=\tan \left(\frac{\psi}{4}\right) \exp \left( \pm \frac{y}{\xi}\right)
$$


where the soliton is centered at $x_{0}= \pm \xi \ln (\tan (\psi / 4))$. The width of the soliton is

$$
\xi=\sqrt{\frac{\hbar v_{F}}{2 \pi w}}
$$

The energy $E_{S}$ of the soliton defined by Eq. (6) is equal to $4 w \xi^{15,22}$ :

$$
E_{S}=2 \sqrt{\frac{2 \hbar v_{F} w}{\pi}} .
$$

The energy $E_{S}$ can be viewed as the phase ordering temperature. Nevertheless thermodynamic equilibrium is not reached since the phase is frozen at a temperature larger than $E_{S}$ due to collective pinning 21.35.36. At the very low temperature considered here the relevant excitations are local deformations of the CDW in the form of bisolitons.

\section{One impurity: bisolitons}

Minimizing the energy with respect to $\varphi(y)$ leads to the exact expression of the phase profile of the bisoliton associated to an impurity located at position $y_{1}$ with a pinning potential $V_{1}$ :

$$
\tan \left(\frac{\varphi(y)}{4}\right)=\tan \left(\frac{\psi}{4}\right) \exp \left(-\frac{\left|y-y_{1}\right|}{\xi}\right),
$$

where $\psi$ is such that

$$
\sin \left(\frac{\psi}{2}\right)=\frac{\pi V_{1} \xi}{2 \hbar v_{F}} \sin \left(\alpha_{1}+\psi\right),
$$

with $\alpha_{1}=Q y_{1}$. The bisoliton has a decay length given by Eq. (7). There can be several solutions to the matching equation (10), corresponding to metastable states separated by energy barriers ${ }^{22,23}$.

\section{A finite concentration of impurities}

\section{Transition to a $3 D$ density wave glass}

The problem of a finite concentration of impurities was treated in Ref. 12 within dynamical renormalization group (RG) describing a quench from high temperature. The first step was to consider two bisolitons at distance $R$. If $R$ is much larger than $\xi$ then the two bisolitons are independent from each other and have independent dynamics. If $R$ is much smaller than $\xi$ there is a single bisoliton pinned by two impurities, with a longer relaxation time than two independent solitons. The case of intermediate values of $R$ was treated approximatively by an exponential interpolation between the limiting cases $R \ll \xi$ and $R \gg \xi$. In the following we consider a simplified model where the pinning energy is additive for
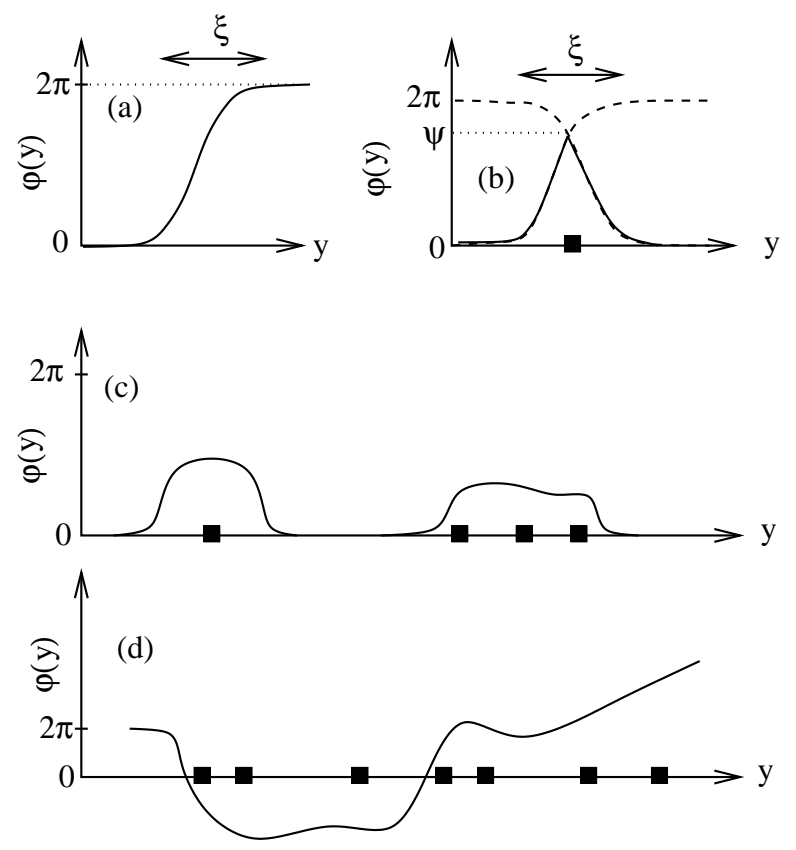

FIG. 1: Schematic representation of the spatial variation of the phase $\varphi(y)$ along a chain. (a) A $2 \pi$ soliton varying over a length $\xi$. (b) A bisoliton 22 (superposition of a $2 \pi$-soliton and a $2 \pi$-anti soliton) varying over a length $\xi$ generated by one strong pinning impurity. (c) Randomly distributed bisolitons generated by the clustering of strong pinning impurities for $x \xi \lesssim 1$. (d) Density wave glass for $x \xi \gtrsim 1$. The filled squares represent the impurities.

all values of $R$ smaller than $\xi$, whereas the two bisolitons are independent from each other for all values of $R$ larger than $\xi$. Considering not only two impurities but a finite concentration of impurities distributed at random in 1D, this defines clusters of impurities: two neighboring impurities at distance $R$ belong to the same cluster if $R<\xi$, and belong to two different clusters if $R>\xi$.

The probability to find $N_{\mathrm{imp}} \geq 1$ impurities in a given cluster follows the exponential distribution

$$
\mathcal{P}\left(N_{\mathrm{imp}}\right)=\exp (-x \xi)[1-\exp (-x \xi)]^{N_{\mathrm{imp}}-1},
$$

where $x$ is the impurity concentration. The average number of impurities within a given cluster is equal to $\exp (x \xi)$. For $x \xi \gtrsim 1$ the number of impurities in a given cluster becomes very large so that the phase is effectively frozen because of large energy barriers. In this regime the system becomes a 3D density wave glass $\frac{15}{15}$ and our treatment (valid if $x \xi \lesssim 1$ ) based on the crude 1D mean field model breaks down.

We suppose in the following that the only $3 \mathrm{D}$ effect is this Larkin-Ovchinikov22.23 level splitting mechanism. We start from the limit of very dilute impurities and increase progressively the concentration. The crude mean field model is expected to describe well localized excitations in the dilute limit $x \xi \lesssim 1$ but at large scale and temperatures higher than the one considered here the system becomes a $3 \mathrm{D}$ elastic medium with different properties 
that we do not discuss in the following. For instance the impurity perturbation decays as a power-law if $D>1$ (see Ref. 24).

\section{Multi-impurity energy landscape}

We suppose that the phase is almost constant and equal to $\psi$ in the middle of a bisoliton and note $X \equiv$ $\tan (\psi / 4)$. The elastic energy corresponding to the second term of Eq. (5) is independent on the number $N_{\text {imp }}$ of impurities because it arises from the two sides of the bisoliton profile where $\partial \varphi(y) / \partial y$ is important. The pinning energy is additive because each impurity brings its own pinning energy.

The energy landscape of a cluster of $N_{\text {imp }}$ impurities generalizing Ref. 12 is given by

$$
\begin{aligned}
& E(X)=16 w \xi \frac{X^{2}}{1+X^{2}} \\
& -2 \sum_{i=1}^{N_{\text {imp }}} V_{i}\left[\sin \left(\frac{\alpha_{i}}{2}\right) \frac{1-X^{2}}{1+X^{2}}+\cos \left(\frac{\alpha_{i}}{2}\right) \frac{2 X}{1+X^{2}}\right]^{2}
\end{aligned}
$$

where the elastic and commensurate energies in the first term are much smaller in magnitude than the pinning energy, but play a role in lifting the degeneracy of the effective two level system.

To illustrate Eq. (12) we show that minimizing with respect to $X$ for impurities at a distance much smaller than $\xi$ is equivalent to solving the sine-Gordon equation in the presence of the pinning term. Let us consider $N_{\mathrm{imp}}$ impurities at positions $y_{1}, \ldots, y_{N_{\mathrm{imp}}}$ and denote by $\psi_{1}, \ldots$, $\psi_{N_{\mathrm{imp}}}$ the value of the phase at the points $y_{1}, \ldots, y_{N_{\mathrm{imp}}}$. The solution with the appropriate boundary conditions is

$$
\tan \left(\frac{\varphi(y)}{4}\right)=\tan \left(\frac{\psi_{1}}{4}\right) \exp \left(\frac{y-y_{1}}{\xi}\right)
$$

for $y<y_{1}$, and

$$
\tan \left(\frac{\varphi(y)}{4}\right)=\tan \left(\frac{\psi_{1}}{4}\right) \exp \left(-\frac{y-y_{N_{\mathrm{imp}}}}{\xi}\right)
$$

for $y>y_{N_{\mathrm{imp}}}$. The derivative of $\varphi(y)$ is discontinuous at the position of the impurities:

$$
\frac{\partial \varphi}{\partial y}\left(y_{k}^{+}\right)-\frac{\partial \varphi}{\partial y}\left(y_{k}^{-}\right)=\frac{2 \pi V_{k}}{\hbar v_{F}} \sin \left(\alpha_{k}+\psi_{k}\right) .
$$

Assuming that $\psi$ is almost constant we obtain

$$
\sin \left(\frac{\psi}{2}\right)=\frac{\pi \xi}{\hbar v_{F}} \sum_{k=1}^{N_{\mathrm{imp}}} V_{k} \sin \left(\alpha_{k}+\psi\right),
$$

generalizing Eq. (10). Solving $\partial E(X) / \partial X=0$ with $E(X)$ given by Eq. (12) and $X=\tan (\psi / 4)$ leads directly to Eq. (16).

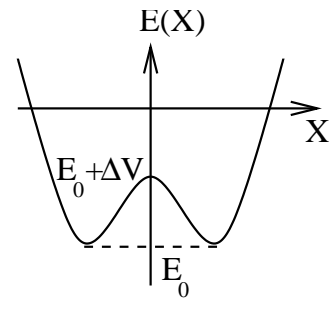

(a)

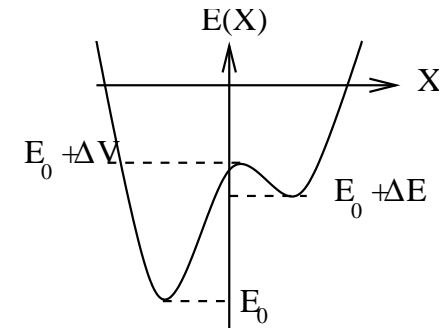

(b)
FIG. 2: Schematic representation of the energy landscape in the commensurate case (a) and in the incommensurate case (b). The ground state is at energy $E_{0}$. The metastable state is at energy $E_{0}+\Delta E$, where $\Delta E$ is the splitting. The unstable "bounce" state is at energy $E_{0}+\Delta V$ where $\Delta V$ is the energy barrier.

\section{E. Properties of the energy landscape}

The energy landscape $E(X)$ given by (12) describes a ground state, separated from a metastable state by an energy barrier. There might be more than two energy minima for some realizations of disorder ${ }^{22.23}$. In this case we restrict the energy landscape to the ground state and to the energy minimum separated from the ground state by the lowest energy barrier. We note $\Delta E$ the difference between the energies of the metastable state and the ground state and $\Delta V$ the energy barrier (see Fig. (2).

\section{Commensurate case}

In the dimerized case $(Q=\pi, \alpha=0) \Delta E$ is equal to zero since the energy landscape is symmetric under a change of sign of the "coordinate" $X$. This can be seen from Eq. (12) by noting that $\cos \left(\alpha_{i} / 2\right)= \pm 1$, $\sin \left(\alpha_{i} / 2\right)=0$ if $Q x_{i}=2 \pi n$, with $n$ an integer, and $\cos \left(\alpha_{i} / 2\right)=0, \sin \left(\alpha_{i} / 2\right)= \pm 1$ if $Q x_{i}=(2 n+1) \pi$, with $n$ an integer. The degeneracy can also be seen directly from Eq. (16) that, in the commensurate case $Q=\pi$, becomes

$$
\cos \left(\frac{\psi}{2}\right)=\left[\frac{2 \pi \xi}{\hbar v_{F}} \sum_{k=1}^{N_{\mathrm{imp}}} V_{k} \cos \alpha_{k}\right]^{-1} .
$$

If $\psi$ is a solution then $-\psi$ is also a solution, explaining the degeneracy. The degeneracy can be also understood by noting that the transformation $\varphi(x) \rightarrow-\varphi(x)$ is a symmetry of the pure system, that is preserved by the pinning term for commensurate impurities but not for incommensurate impurities.

In the commensurate case it is thus not possible to communicate energy over long time scales to the effective two-level system by increasing temperature. The classical model predicts no slow relaxation at all in the commensurate case whereas in experiments there exists slow 


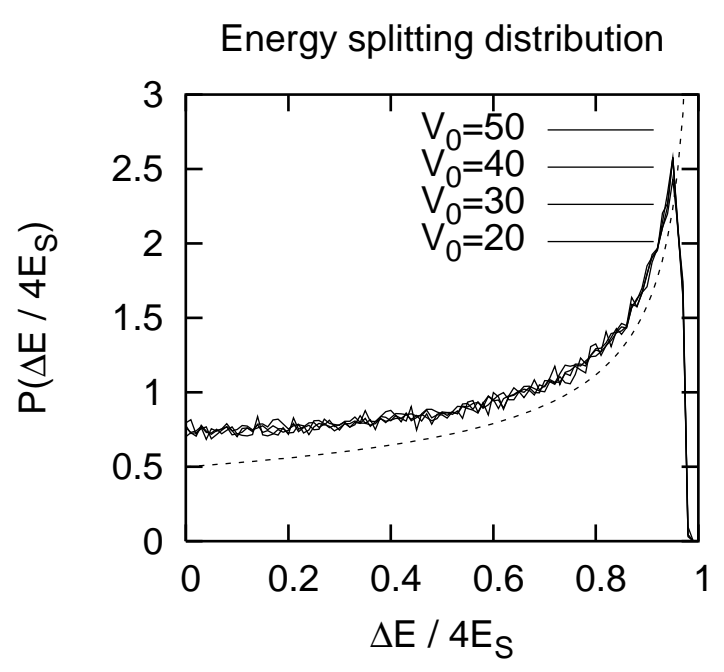

FIG. 3: Distribution of level splitting $\Delta E$ for the model with random phases discussed in section IID The impurities are distributed at random on a chain of length $\xi$. The impurity concentration is such that the average number of impurities is $\bar{N}_{\mathrm{imp}}=x \xi=1$. The curves corresponding to $x \xi=0.5$ and $x \xi=5$ are superposed on the curves corresponding to $x \xi=1$. The pinning potentials $V_{i}$ are uniformly distributed in the interval $\left[0, V_{0}\right]$ with $V_{0}$ indicated on the figure. The distribution of level splitting normalized to $4 E_{S}$ is almost independent on $w \xi$ and $V_{0}$, chosen such that $4 E_{S} \ll V_{0}$. The dashed line corresponds to $P(z)=1 /[2 \sqrt{1-z}]$, with $z=\Delta E / 4 E_{S}$ (see Eq. [18).

relaxation, even though faster than in the incommensurate compound 29 . Adding quantum tunneling between the two energy minima of the energy landscape can generate two non degenerate energy levels corresponding to symmetric and antisymmetric wave functions, therefore restoring a finite heat response.

\section{Energy splitting distribution in the incommensurate case}

The distribution of energy splitting is shown on Fig. 3 The distribution of splitting is close to

$$
P\left(\frac{\Delta E}{4 E_{S}}\right) \simeq \frac{1}{2}\left(1-\frac{\Delta E}{4 E_{S}}\right)^{-1 / 2} .
$$

The most probable level spacing is close to $4 E_{S}$, independent on the value of the pinning potential and on the number of impurities involved in the bisoliton, showing that the shape of $P\left(\Delta E / 4 E_{S}\right)$ is almost unchanged when the concentration of impurities increases. The energy splitting distribution (18) can be understood in the case of a single soliton by noting that the pinning energy is much larger than the elastic energy so that the elastic term can be treated as a perturbation. There are four values of $X$ minimizing the pinning energy:

$$
X_{0}^{(\epsilon)}=\frac{\epsilon-\sin (\alpha / 2)}{\cos (\alpha / 2)}
$$

$$
X_{1}^{(\epsilon)}=\frac{\epsilon+\cos (\alpha / 2)}{\sin (\alpha / 2)},
$$

with $\epsilon= \pm 1$. The pinning energy of the solution (19) is $E_{\text {pin }}\left(X_{0}^{(\epsilon)}\right)=-2 V$, and the pinning energy of the solution (20) is $E_{\text {pin }}\left(X_{1}^{(\epsilon)}\right)=0$. The elastic energy of the solution $X_{0}^{(\epsilon)}$ is $E_{\mathrm{el}}\left(X_{0}^{(\epsilon)}\right)=2 E_{S}(1-\epsilon \sin (\alpha / 2))$. The energy splitting $E_{\mathrm{el}}\left(X_{0}^{(-)}\right)-E_{\mathrm{el}}\left(X_{0}^{(+)}\right)$is then distributed according to Eq. (18) since $\alpha$ is uniformly distributed.

The existence of the upper bound $4 E_{S}$ in the energy splitting distribution is compatible with the experimental observation of a high temperature tail $C_{v} \sim 1 / T^{2}$ of a Schottky anomaly in the equilibrium specific heat, with a well-defined level splitting. Experimentally the level splitting $\Delta E_{0}$ of a two-level system is related to the temperature $T_{\max }$ of the maximum of the Schottky anomaly by the relation $\Delta E_{0} \simeq 2.5 k_{B} T_{\max }$. It was shown experimentally that $T_{\max }<30 \mathrm{mK}^{9}$ so that $\Delta E_{0}=4 E_{S} \lesssim$ $100 \mathrm{mK}$. The existence of a well-defined level splitting in experiments is a universal property, valid for commensurate and incommensurate systems, and independent on the value of the CDW or SDW critical temperature that can vary by more than one order of magnitude from one compound to the other $\left(T_{\text {Peierls }}=218 \mathrm{~K}\right.$ for $\mathrm{o}-\mathrm{TaS}_{3}$, $T_{\mathrm{SDW}}=12 \mathrm{~K}$ for $(\mathrm{TMTSF})_{2} \mathrm{PF}_{6}, T_{\mathrm{SP}}=15 \mathrm{~K}$ for the spin-Peierls compound (TMTTF) ${ }_{2} \mathrm{PF}_{6}, T_{\mathrm{AF}}=13 \mathrm{~K}$ for the antiferromagnet (TMTTF $)_{2} \mathrm{Br}$ ). This is compatible with the fact that $\Delta E_{0}$ is related only to the strength $w$ of interchain interactions and the Fermi velocity $v_{F}$ :

$$
\Delta E_{0}=4 E_{S}=\frac{16}{\sqrt{2 \pi}} \sqrt{\hbar v_{F} w}
$$

where $v_{F}$ has the same magnitude for several compounds (for instance $v_{F}=0.86 \times 10^{7} \mathrm{~cm} \cdot \mathrm{sec}^{-1}$ in $\left.(\mathrm{TMTSF})_{2} \mathrm{PF}_{6}{ }^{37}\right)$ and interchain couplings are also expected to take similar orders of magnitude, from what we deduce that $\Delta E_{0}$ is almost identical in all samples, independent on the charge or spin gap. The energy $\Delta E_{0}$ is proportional to the elastic energy $E_{S}$ of a $2 \pi$-soliton in the absence of impurities, not to be confused with the activation energy $E_{A}$ of the order of $100 \mathrm{~K}$ probed in transport experiments ${ }^{38}$. There is thus no inconsistency in the difference between the orders of magnitude of $E_{A}$ and $E_{S}$.

\section{Distribution of energy barriers}

The distribution of energy barriers $P\left(\Delta V / V_{0}\right)$ is well fitted by an exponential function (see Fig. 4). The average relaxation time defined as

$$
\tau_{0} \int d(\Delta V) P(\Delta V) \tau_{0} \exp (\Delta V / T),
$$

with $T$ the temperature diverges at a finite temperature, like in the REM-like trap model ${ }^{11}$. However this model, useful for discussing waiting time effects, over evaluates 


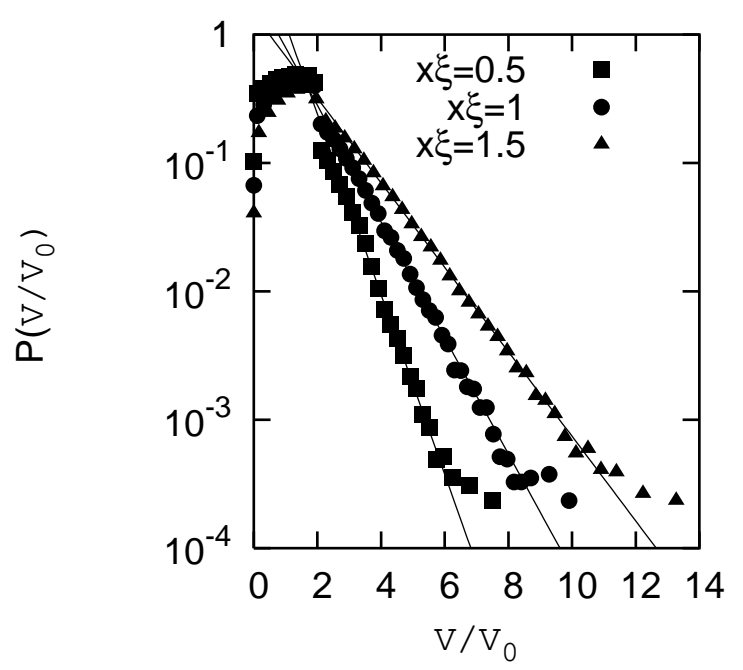

FIG. 4: Distribution of the dimensionless energy barrier $\Delta V / V_{0}$ in the cluster model. We use $x \xi=0.5,1,1.5$, $k_{F}=\pi / 2+0.02$. The pinning potentials $V_{i}$ are uniformly distributed in the interval $\left[0, V_{0}\right]$ with $V_{0}=50$. The solid lines correspond to the fits $P\left(\Delta V / V_{0}\right)=1.45 \times$ $\exp \left(-0.76 \times \Delta V / V_{0}\right)$ for $x \xi=0.5, P\left(\Delta V / V_{0}\right)=2.25 \times$ $\exp \left(-1.04 \times \Delta V / V_{0}\right)$ for $x \xi=1$, and $P\left(\Delta V / V_{0}\right)=6 \times$ $\exp \left(-1.61 \times \Delta V / V_{0}\right)$ for $x \xi=1.5$.

glassiness compared to the dynamical RG already discussed in Ref. 12 (see section IG).

\section{F. Out-of-equilibrium specific heat}

To evaluate energy relaxation in the incommensurate case, we restrict the energy landscape to the ground state, the metastable state and the unstable "bounce" state at the top of the barrier. We are left with a two-state trap model with energies $-E_{1}$ and $-E_{2}$ (with $E_{1}>E_{2}$ ). $E_{1}$ is equal to the barrier $\Delta V$ calculated in the preceding section and $E_{2}$ is equal to the barrier $\Delta V$ minus the splitting $\Delta E$. The unstable state is at zero energy. We note $P_{1}(t)$ $\left(P_{2}(t)\right)$ the probability to be in state "1" ("2") at time $t$ and note $\tilde{P}_{1,2}(t)=P_{1,2}(t) \exp \left(-E_{1,2} / 2 T\right)$. The evolution of the probabilities is given by Glauber dynamics ${ }^{39}$

$$
\tau_{0} \frac{d}{d t}\left[\begin{array}{c}
\tilde{P}_{1} \\
\tilde{P}_{2}
\end{array}\right]=\hat{G}\left[\begin{array}{c}
\tilde{P}_{1} \\
\tilde{P}_{2}
\end{array}\right]
$$

with $G_{1,1}=-\exp \left(-E_{1} / T\right), G_{2,2}=-\exp \left(-E_{2} / T\right)$, $G_{1,2}=G_{2,1}=\exp \left(-\left(E_{1}+E_{2}\right) / 2 T\right)$. Experimentally the time $\tau_{0}$ associated to bisoliton dynamics is of order of $1 \mathrm{sec}$ for the thermally activated process. The time dependence of the occupation probabilities $P_{1}(t)$ and $P_{2}(t)$ is obtained by diagonalizing the $2 \times 2$ matrix $\hat{G}$, from what we deduce the value of the energy $U\left(t_{w}, t_{w}+\tau, T\right)$ as a function of the waiting time $t_{w}$, the time $\tau$ elapsed since the waiting time and temperature $T$. The out-ofequilibrium total specific heat is defined as the total heat

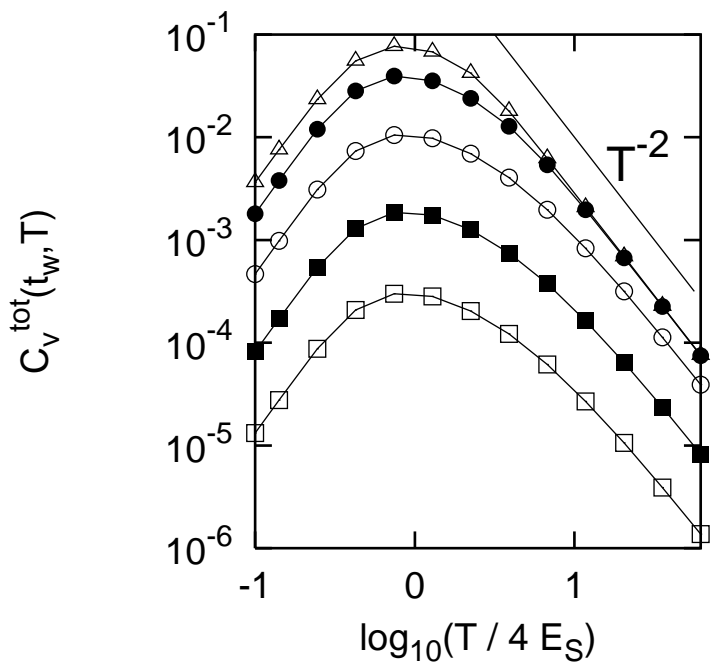

FIG. 5: Variation of the out-of-equilibrium total specific heat as a function of $\log _{10}\left(T / 4 E_{S}\right)$, for different values of $t_{w} / \tau_{0}: \log _{10}\left(t_{w} / \tau_{0}\right)=-2(\square), \log _{10}\left(t_{w} / \tau_{0}\right)=-1.2$ $(\mathbf{\square}), \log _{10}\left(t_{w} / \tau_{0}\right)=-0.4(\mathrm{o}), \log _{10}\left(t_{w} / \tau_{0}\right)=0.4(\bullet)$. $\log _{10}\left(t_{w} / \tau_{0}\right)=1.2(\triangle)$. We used $x \xi=1$. The pinning potentials $V_{i}$ are uniformly distributed in the interval $\left[0, V_{0}\right]$ with $V_{0} / E_{S}=12.5$. The specific heat for long $t_{w} / \tau_{0}$ follows a $1 / T^{2}$ behavior for $T / 4 E_{S} \gtrsim 1$, where $E_{S}$ is the energy of a soliton. The solid line represents the $C_{v} \sim T^{-2}$ behavior.

released divided by the temperature variation:

$$
C_{v}^{\text {tot }}\left(t_{w}, T\right)=\frac{U\left(t_{w}, t_{w}, T+\Delta T\right)-U(0,0, T)}{\Delta T} .
$$

The dynamics equations can be solved exactly in the case of a single two-level system with energies $-E_{1}$ and $-E_{2}$ and expanded in the high temperature regime $T \gg \mid E_{1}-$ $E_{2} \mid$, leading to the specific heat

$$
\begin{aligned}
C_{v} & =\frac{\left(E_{1}-E_{2}\right)^{2}}{4 T^{2}} \\
& \times\left[1-\exp \left[-\frac{t_{w}}{\tau_{0}}\left[\exp \left(-\frac{E_{1}}{T}\right)+\exp \left(-\frac{E_{2}}{T}\right)\right]\right]\right],
\end{aligned}
$$

proportional to $1 / T^{2}$, as expected for the high temperature tail of a Schottky anomaly. Increasing the waiting time increases the energy transfered to the two-level system and therefore increases the amplitude of the $1 / T^{2}$ term.

The variations of the out-of-equilibrium total specific heat $C_{v}^{\mathrm{tot}}\left(t_{w}, T\right)$ as a function of temperature $T$ for different values of the waiting time $t_{w}$ are shown on Fig. 5 for a finite concentration of incommensurate impurities. At temperatures larger than the maximal splitting $\Delta E=4 E_{S}$ the out-of-equilibrium specific heat follows a $1 / T^{2}$ behavior. The out-of-equilibrium specific heat is strongly reduced as the waiting time decreases but still follows a $1 / T^{2}$ behavior, in a qualitative agreement with experiments (see Fig. 3 in Ref. 9). 


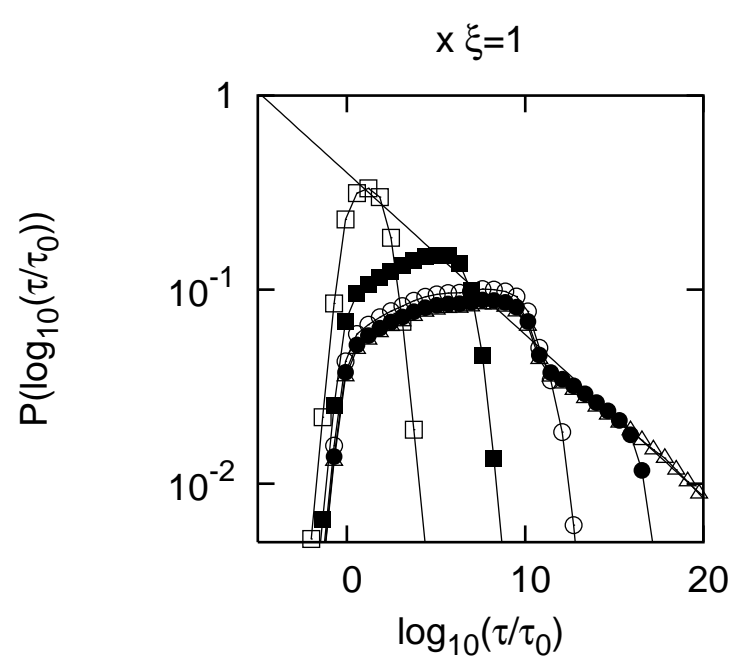

FIG. 6: Spectrum of relaxation times deduced from energy relaxation (see Eq.(27)). We use $V_{0} / 4 E_{S}=12.5, T / 4 E_{S}=400$, and $x \xi=1$. The pinning potentials $V_{i}$ are uniformly distributed in the interval $\left[0, V_{0}\right]$. The waiting times correspond to $\log _{10}\left(t_{w} / \tau_{0}\right)=2.4(\square), 7.2(\mathbf{\square}), 12(\bullet), 16.8(\circ), 21.6(\triangle)$. The solid line is a fit to $\log _{10}\left(P\left(\log _{10} \tau\right)\right)=a+b \log _{10} \tau$, with $b=-0.083$.

\section{G. Spectrum of relaxation times}

Let $U\left(t_{w}, t_{w}+\tau, T\right)$ be the energy of the two-level system at time $t=t_{w}+\tau$, with a heat pulse applied between $t=0$ and $t=t_{w}$. The spectrum of relaxation times is deduced from $U\left(t_{w}, t_{w}+\tau, T\right)$ by assuming that

$$
U\left(t_{w}, t_{w}+\tau, T\right)=\int P_{t_{w}}\left(\ln \tau^{\prime}\right) \exp \left(-\tau / \tau^{\prime}\right) d \ln \tau^{\prime}
$$

Replacing the exponential by a step-function leads to the relaxation time spectrum 1

$$
P_{t_{w}}(\ln \tau) \propto \frac{\partial U\left(t_{w}, t_{w}+\tau, T\right)}{\partial \ln \tau} .
$$

We have shown on Fig. [6 the spectra of relaxation times $P_{t_{w}}(\ln \tau)$ for $x \xi=1$. Similar results are obtained for $x \xi=0.5$ and $x \xi=1.5$, with however a different value of the exponent of the power-law. The long time tail of the spectrum is a power-law, in agreement with experiments (see Ref. 12). As the waiting time increases the power-law regime extends to ever longer times without limits, compatible with the existence of a genuine glass transition that is an artifact of this clustering model.

For comparison we have also calculated the relaxation time spectra obtained from dynamical RG (see Fig. (7). The calculation is identical to Ref. 12: we suppose a quench from high temperature at time $t=0$. The small time degrees of freedom are progressively eliminated, making bigger object that relax more slowly. In this approach the energy landscape of two impurities at distance $R$ is interpolated between the two limiting cases

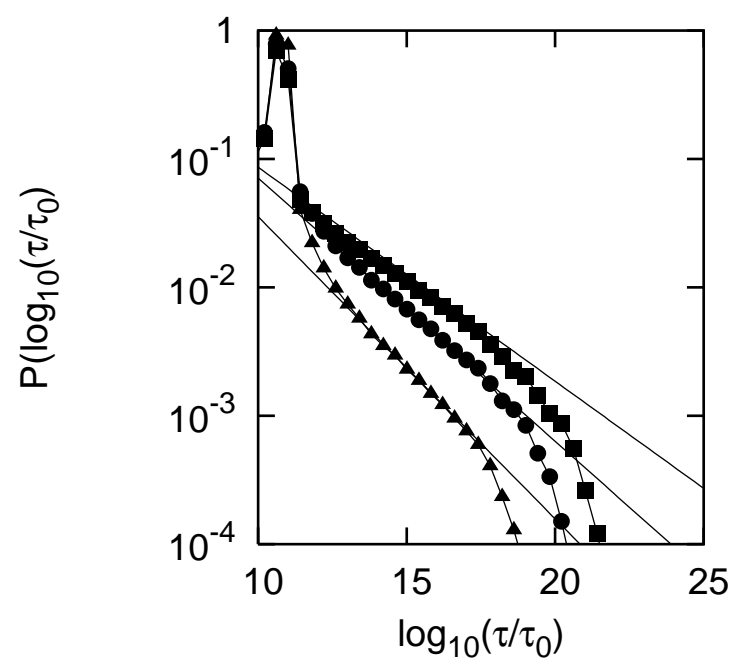

FIG. 7: Spectrum of relaxation times deduced from dynamical renormalization group. We use $V_{0} / 4 E_{S}=12.5$, $T / 4 E_{S}=400$, , and $x \xi=1.5(\boldsymbol{\square}), x \xi=1(\bullet)$ and $x \xi=0.5$ $(\boldsymbol{\Delta})$. The pinning potentials $V_{i}$ are uniformly distributed in the interval $\left[0, V_{0}\right]$. The solid line is a fit to $\log _{10}\left(P\left(\log _{10} \tau\right)\right)=$ $a+b \log _{10} \tau$, with $b=-0.23$ for $x \xi=1.5, b=-0.21$ for $x \xi=1$ and $b=-0.17$ for $x \xi=0.5$. We carried out simulations with larger system sizes and found practically no finite size effects on the data on this figure.

$R \ll \xi$ and $R \gg \xi$ so that the phase fluctuations are more important compared to the previous clustering model. Impurities can thus be depinned in sequence, not necessarily all together. We obtain also a power-law relaxation but (i) the exponent of the power-law spectrum of relation times is different from the clustering model (smaller relaxation times are favored), and (ii) ageing is "interrupted" for the clustering model (there exists a maximal relaxation time). As expected the clustering model discussed previously over evaluates glassiness but is nevertheless useful for addressing qualitatively waiting time effects that could not be discussed within dynamical RG. Experiments favor interrupted ageing with respect to a genuine dynamical glass transition (see Ref. 12 for an analysis of experiments based on a REM-like model that is justified microscopically by the dynamical RG approach).

\section{QUANTUM LIMIT (SUBSTITUTIONAL AND STRONG PINNING IMPURITIES)}

\section{A. Preliminaries}

We base the discussion in this section on the electronic part of the Peierls Hamiltonian. We discuss a model of disordered CDW there is only a charge sector, or a model of disordered SDW where there is only a spin sector. We expect this simplified models to capture part of the properties of real compound in which there is a gap in both 
(a)

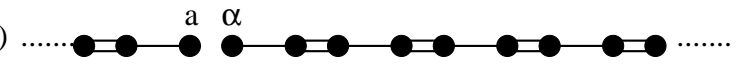

(b)

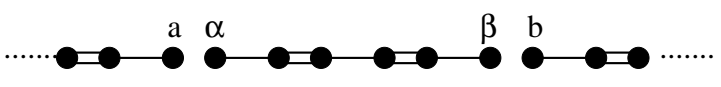

(c)

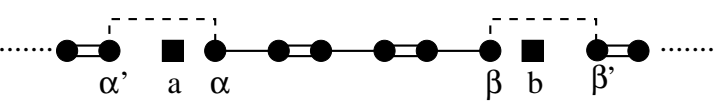

(d)

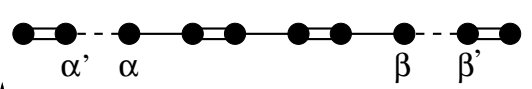

(e)

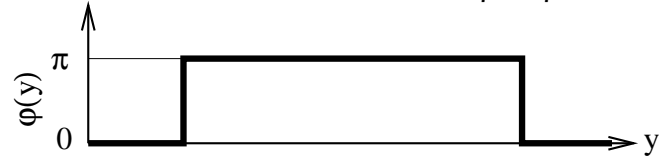

FIG. 8: Schematic representation of a dimerized CDW chains with open boundary conditions with one impurity (a) or two impurities (b). The double lines represent the strong bonds $t+$ $\epsilon$. (b) corresponds to $R=y_{\beta}-y_{\alpha}$ odd. We represented on (c) two substitutional impurities (black square) leaving unpaired fermions at sites $\alpha$ and $\beta$. (d) corresponds to the chain where the impurity sites have been removed ${ }^{34}$. (e) shows the spatial variation of the phase corresponding to the chain (d).

the spin and charge channels. The analogy between the CDW case with only a charge channel and the SDW case with only a spin channel is based on the fact that one dimensional tight binding models can be mapped onto XX magnets in one dimension through a Jordan-Wigner transformation. It was shown explicitely in the dimerized case that the properties of substitutional disorder are almost identical for the XX and Heisenberg models 34 in spite of interactions between Jordan-Wigner fermions in the Heisenberg case. We expect this similarity to be valid also in the incommensurate case.

Quantum effect were already discussed in Ref. 21 but here we work within a different microscopic model that was proved to be useful in the case of doped spin-Peierls systems $^{34}$, and generalize it to the case of incommensurate CDWs. Substitutional disorder in a spin-Peierls system (relevant to $\mathrm{Cu}_{1-x} \mathrm{Zn}_{x} \mathrm{GeO}_{3}{ }^{30.31 .32 .33}$ ) is qualitatively equivalent to impurities that break the chains into finite length segments (see Ref. 34 and section IICC). We have shown on Fig. 8 a schematic representation of substitutional impurities in dimerized chains. The phase of the dimerization changes by $\pi$ in the chain where the site of the impurity has been removed. For a CDW or SDW with a wave-vector $Q=2 k_{F}$ the phase changes by $Q a_{0}$ once the impurity sites have been removed, where $a_{0}$ is the lattice parameter. The absolute value of the amplitude of the CDW is supposed to remain constant. The solitons generated by the substitutional disorder that we consider here are thus phase solitons, connecting two degenerate ground states with different phases. One can cross-over continously from edge states in the limits $t_{\alpha, \alpha^{\prime}}=t_{\beta, \beta^{\prime}}=0$ to solitons connecting two ground states as the parameters $t_{\alpha, \alpha^{\prime}}$ and $t_{\beta, \beta^{\prime}}$ are increased.

There is a flat classical energy landscape in the case of open chains for the model defined by Eq.(5) in the limit $w=0$. The classical Hamiltonian is just (5) on an open chain, without the pinning term:

$$
\begin{aligned}
\mathcal{H} & =\frac{\hbar v_{F}}{4 \pi} \int_{0}^{L} d y\left(\frac{\partial \varphi(y)}{\partial y}\right)^{2} \\
& +w \int_{0}^{L} d y[1-\cos \varphi(y)]
\end{aligned}
$$

that has no bisoliton metastable states like in the preceding section (the ground state is $\varphi(y)=0$ ). The bound state associated to a single substitutional impurity in the quantum model defined by the Peierls Hamiltonian

$$
\mathcal{H}=\sum_{i}\left[t+\epsilon \cos \left(2 k_{F} y_{i}\right)\right]\left[c_{i+1}^{+} c_{i}+c_{i}^{+} c_{i+1}\right]
$$

is thus at the lowest possible energy (exactly in the middle of the gap). The variable $t$ in Eq. (29) is equal to the average hopping amplitude and $\epsilon$ is the amplitude of the modulation. The variable $y_{i}$ in Eq.(29) is the coordinate of the site number $i: y_{i}=i a_{0}$, with $a_{0}$ the lattice parameter. The bound state due to substitutional disorder can be occupied by an electron and a hole with an equal probability, and there is a fast dynamics of the occupation probability since there are no energy barriers in the classical limit. Like in spin glasses mostly governed by RKKY interactions it is expected that the "microscopic time" $\tau_{0}$ is extremely small $\left(\tau_{0} \simeq 10^{-12} \mathrm{sec}\right.$ in spin glasses). By contrast there is slow dynamics with $\tau_{0} \simeq 1$ sec in the case of the organic spin-Peierls compound (TMTTF) $)_{2} \mathrm{PF}_{6}$, well described by the classical model discussed previously.

The width of a soliton in the quantum limit is equal to

$$
\xi_{0}(E)=\frac{2 \hbar v_{F}}{\sqrt{\Delta^{2}-E^{2}}}
$$

where $E$ is the energy, $\Delta$ is equal to the Peierls gap and $v_{F}$ is the Fermi velocity in the absence of lattice distortions. $\xi_{0}$ is different from the soliton or bisoliton width in the classical limit $\xi=\sqrt{\hbar v_{F} / 2 \pi w}$ discussed in the preceding section. In the classical model one should incorporate interchain interactions for the soliton or bisoliton to have a finite width whereas in the quantum model the zero energy soliton width is finite in the absence of interchain interactions. Experimentally the slow relaxation properties associated to the $1 / T^{2}$ tail of the specific heat are independent on the value of the gap $\Delta$ and of the transition temperature that can vary by more than one order of magnitude. In the case of the SDW (TMTSF) ${ }_{2} \mathrm{PF}_{6}$ the BCS-like relation $\Delta \simeq 1.7 T_{c}$ is well verified $\left(T_{c}=11.5 \div 12 \mathrm{~K}\right.$ and $\Delta=20 \mathrm{~K}$ from $\left.\mathrm{NMR}^{40}\right)$. The Fermi velocity ${ }^{37}$ is $v_{F} \simeq 0.86 \times 10^{7} \mathrm{~cm} \cdot \mathrm{sec}^{-1}$ so that $\xi_{0}=2 \hbar v_{F} / \Delta \simeq 90 a_{0}$, with the lattice parameter $a_{0}=7.3 \AA$. In the case of o- $\mathrm{TaS}_{3}$ we do not have a precise data for $v_{F}$ and we 
take $v_{F}=10^{7} \mathrm{~cm} \cdot \mathrm{sec}^{-1}$ as a typical value. We have $T_{c}^{(\mathrm{CDW})}=215 \mathrm{~K}$ and $\Delta_{\mathrm{CDW}}=3.73 T_{c} \simeq 780 \mathrm{~K}$ so that $\xi_{0}^{(\mathrm{CDW})}=6 a_{0}$ with $a_{0}=3.34 \AA$ A. However it is likely that there is also a smaller spin gap since the spin susceptibility follows a power-law. An energy scale $E=30 \mathrm{~K}$ can be estimated from the deviations of the power-law. Assuming that the power-law susceptibility is due to randomly distributed magnetic moments we identify the energy scale $E$ to the average exchange energy $J_{\mathrm{av}}=\Delta x \xi$ with $x \xi=0.3$ estimated from the power-law susceptibility (see section IIIC2) so that $\xi_{0}^{\text {(spin) }}=150 \div 450 a_{0}$. $\xi_{0}^{\text {(spin) }}$ in the SDW compound (TMTSF) ${ }_{2} \mathrm{PF}_{6}$ and o- $\mathrm{TaS}_{3}$ are thus one order of magnitude larger than $\xi_{0}$ in the spin-Peierls compound $\mathrm{CuGeO}_{3}$.

\section{B. Strong pinning impurities}

\section{Dyson matrix}

The quantum model given by Eq. (29) can also be used to treat strong pinning impurities, not only substitutional disorder. The Hamiltonian is equal to (29), plus a term describing the impurity potential:

$$
H_{\mathrm{imp}}=-\sum_{i} V_{y_{i}} c_{y_{i}}^{+} c_{y_{i}}
$$

where the strong pinning impurities are located at random positions $\left\{y_{1}, \ldots, y_{\mathrm{N}_{\mathrm{imp}}}\right\}$. We suppose that $V_{y_{i}}=V$ is the same for all impurities and that $V>0$. We note $G(E)$ the Green's function at energy $E$ in the presence of the pinning potential and $g(E)$ the Green's function in the absence of pinning potential. The Green's function $G(E)$ is obtained from inverting the Dyson matrix:

$$
\sum_{k=1}^{N_{\mathrm{imp}}}\left[\delta_{i, k}+g_{y_{i}, y_{k}}(E) V_{y_{k}}\right] G_{y_{k}, y_{j}}(E)=g_{y_{i}, y_{j}}(E),
$$

where the Green's function $g_{y_{i}, y_{k}}(E)$ is given in Appendix A The bound state energies correspond to the poles of $G(E)$.

\section{Two pinning centers}

In the commensurate case $k_{F} a_{0}=\pi / 2$ the energy levels evolve smoothly as a function of the distance between the two impurities (see Fig. 94(a)). In the incommensurate case $k_{F} a_{0}=\pi / 2+\delta k_{F}$ (see Fig. 9.(b)) the energy levels fluctuate strongly as the distance between the two impurities is reduced. This shows that quantum mechanical interactions among impurities enhance disorder effects in the incommensurate case but not in the commensurate case.
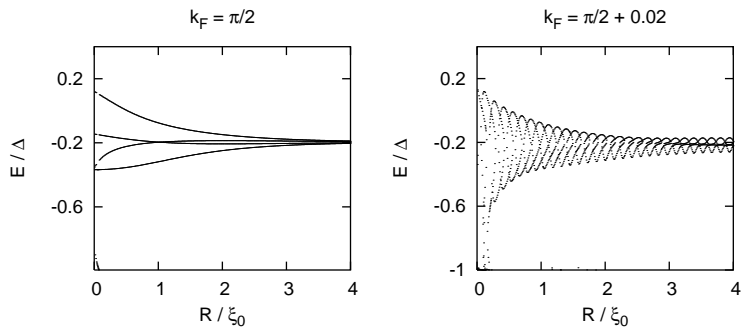

FIG. 9: Evolution of the bound state energy levels as a function of the distance between the two pinning centers, in the commensurate case $k_{F}=\pi / 2(\mathrm{a})$ and in the incommensurate case $k_{F}=\pi / 2+\delta k_{F}$, with $\delta k_{F}=0.02(\mathrm{~b})$. The energy is in units of $\Delta=\sqrt{2} \epsilon$ and the distance is in units of $\xi_{0}=2 v_{F} / \Delta$. We used $\epsilon / t=10^{-3}$ and $V / t=10$.

\section{Substitutional disorder}

\section{Open chains in the dimerized limit}

Let us consider two semi-infinite dimerized chains ending at sites "a" and " $\alpha$ " (see Fig. 8.(a)). We note $t_{0}=t_{a, \alpha}$ the value of the hopping between sites "a" and " $\alpha$ " (equal to $t+\epsilon$ or $t-\epsilon$ ) in the infinite chain. The Dyson equation relates the Green's functions $g_{i, j}$ of the infinite chain to the Green's functions $G_{i, j}$ of the semiinfinite chain:

$$
\begin{aligned}
g_{a, a}(E) & =G_{a, a}(E)+t_{0}^{2} G_{a, a}(E) g_{a, a}(E) \\
g_{\alpha, \alpha}(E) & =G_{\alpha, \alpha}(E)+t_{0}^{2} G_{a, a}(E) g_{\alpha, \alpha}(E),
\end{aligned}
$$

with $g_{a, a}(E)=g_{\alpha, \alpha}(E)=E /\left(2 t \sqrt{2 \epsilon^{2}-E^{2}}\right)$. The solution of (33) and (34) is

$$
G_{a, a}(E)=\frac{t}{t_{0}^{2}} \frac{\sqrt{2 \epsilon^{2}-E^{2}}}{E}\left\{-1+\eta_{a} \sqrt{1+\frac{t_{0}^{2}}{t^{2}} \frac{E^{2}}{2 \epsilon^{2}-E^{2}}}\right\},
$$

with $\eta_{a}= \pm 1$. In the case of two weak bonds in the chain (see Fig. 8.(b)) we find $G_{\alpha, \beta}=g_{\alpha, \beta} / \mathcal{D}$, with

$$
\begin{aligned}
\mathcal{D} & =\left[1+t_{a, \alpha}^{2} G_{a, a} g_{\alpha, \alpha}\right]\left[1+t_{b, \beta}^{2} G_{b, b} g_{\beta, \beta}\right] \\
& -t_{a, \alpha}^{2} t_{b, \beta}^{2} G_{a, a} G_{b, b} g_{\alpha, \beta} g_{\beta, \alpha} .
\end{aligned}
$$

If the separation between the boundaries is large enough the energy of the bound states can be expanded in the small parameter $\exp \left(-R / \xi_{0}\right)$ where $\xi_{0} / a_{0}=2 \sqrt{2} t / \epsilon$ is the zero energy coherence length. Since the bound states are close to the Fermi energy we also expand $G_{\alpha, \beta}$ in powers of $E$. If $R=y_{\beta}-y_{\alpha}$ is even we find that one bound state is generated at one end of the chain. In the case $R$ odd on Fig. 8.(b) we find two bound states at $E^{( \pm)}= \pm(\epsilon / 3) \exp \left(-R / \xi_{0}\right)$ corresponding to $\eta_{a}=\eta_{b}=$ -1 . In the ground state $E^{(-)}$is occupied with an electron and $E^{(+)}$is occupied with a hole. The lowest neutral excitation corresponds to an electron in the level $E^{(+)}$ and a hole in the level $E^{(-)}$. This is in agreement with a 


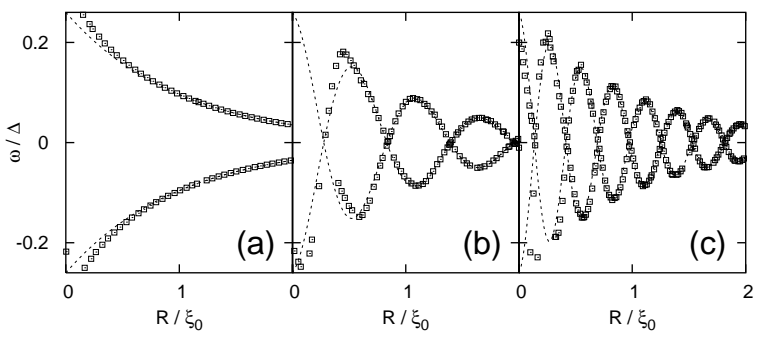

FIG. 10: Evolution of the bound state energies as a function of the distance $R=\left|y_{a}-y_{b}\right|$ between the two impurities. We use $k_{F}=\pi / 2+\delta k_{F}$, with $\delta k_{F}=0$ (a), $\delta k_{F}=0.02$ (b) and $\delta k_{F}=0.04(\mathrm{c})$. We use the parameters $\epsilon / t=10^{-2}$. $t_{\alpha, \alpha^{\prime}} / t=t_{\beta, \beta^{\prime}} / t=1 / 2$. The dashed line is a fit to $E / \Delta=$ $\pm 0.26 \cos \left(\left(\delta k_{F}\right) R\right) \exp \left(-R / \xi_{0}\right)$.

previous model developed for the spin-Peierls compound $\mathrm{Cu}_{1-x} \mathrm{Zn}_{x} \mathrm{O}_{3}{ }^{34}$ as well as in a qualitative agreement with numerical simulations 41.42 .

\section{Substitutional disorder in $C D W s$ and $S D W s$}

We consider now a more realistic model of substitutional disorder, both for commensurate and incommensurate CDWs. We suppose that two substitutional impurities "remove" the sites "a" and "b" from the chain and that second neighbor interactions $t_{\alpha, \alpha^{\prime}}$ and $t_{\beta, \beta^{\prime}}$ couple the right and left neighbors (see Fig. 8 (c)). The model is similar to Ref. 34 proposed for $\mathrm{Zn}$ impurities in $\mathrm{CuGeO}_{3}$ except that we consider here the incommensurate case. We calculate the poles and residues as a function of the distance $R=\left|y_{a}-y_{b}\right|$ between the two impurities (see Fig. 10). We use $k_{F}=k_{F}^{(0)}+\delta k_{F}$, with $k_{F}^{(0)}=\pi / 2$ and show on Fig. 10 the Friedel oscillations of the bound state levels for odd values of $R$. The phase of the Friedel oscillations is shifted by $\pi / 2$ for even values of $R$. We deduce from the level crossings that there exits a change of sign in the hopping amplitude between the two solitons as $R$ is increased.

Similarly to a model of doped spin-Peierls system ${ }^{34}$ substitutional disorder in CDWs and SDWs provides an explanation to the power-law specific heat $C_{v} \sim T^{\alpha}$ observed in disordered CDWs and SDWs, and to the susceptibility $\chi(T) \sim T^{-1+\alpha}$ observed in the CDW o- $\mathrm{TaS}_{3}{ }^{27}$ (with the same value of $\alpha$ in the specific heat and susceptibility experiments). In experiments on the SDW (TMTSF $)_{2} \mathrm{PF}_{6}{ }^{10}$, on changing the time constant in a heat pulse experiment by a factor of 100 , there is an increase by a factor of 7 of the amplitude of the $T^{-2}$ contribution whereas the $T^{\alpha}$ contribution changes only by $20 \%$, and not in a systematic manner. In the commensurate (TMTTF) ${ }_{2} \mathrm{Br}$ compound and also in a heat pulse experiment, an increase of the time constant by a factor of 3 results in an increase of the $T^{-2}$ term by a factor of 3 and only an increase of the $T^{\alpha}$ term by $20 \%$. This shows that the $T^{\alpha}$ contribution to the spe- cific heat can be interpreted as an equilibrium property, which is the case if frustration due to Friedel oscillations can be neglected as in a dilute one dimensional model. Focusing on the SDW case we consider only exchanges $J(R)$ between nearest neighbor solitons and disregard the changes of sign in $J(R)$. The exchange distribution scales like $P(|J|) \sim|J|^{-1+x \xi_{0}}$, where $x$ is the concentration of substitutional impurities 34 . The energy of "active" pairs of spins is approximately given by $U=\int_{0}^{T} J P(J) d J$, where $T$ is the temperature so that the temperature dependence of the specific heat is given by $C_{v} \sim T^{x \xi_{0}}$. The susceptibility is approximately given by a Curie contribution for the fraction of "active" spin having an energy smaller than $T$ so that the susceptibility behaves like $\chi(T) \sim T^{-1+x \xi_{0}}$, leading to $\alpha=x \xi_{0}$. Experimentally $\alpha=0.3 \div 1.2$ as mentioned in the Introduction. In the case of o- $\mathrm{TaS}_{3}$ we estimate $\xi_{0} \simeq 150 \div 450 a_{0}$ in the spin sector as mentioned previously and $\alpha=0.3$ obtained from specific heat and susceptibility. We deduce the concentration of intrinsic substitutional impurities $x_{\text {int }} \simeq 0.07 \div 0.2 \%$. The upper bound is comparable to the nominal concentration of extrinsic $\mathrm{Nb}$ impurities $x_{\text {ext }} \simeq 0.5 \%$ which might explain why the exponent $\alpha$ in the experiment is the same in the presence or absence of extrinsic impurities.

\section{CONCLUSIONS}

To conclude we have investigated several factors involved in energy relaxation in disordered CDWs and SDWs. A first factor is the role of commensuration. In the commensurate case we find that the energy landscape is symmetric since the symmetry $\varphi(x) \rightarrow-\varphi(x)$ of the CDW Hamiltonian is preserved by the impurity potential. As a consequence the two energy minima are degenerate, a property that can be obtained by a direct solution of the sine-Gordon equation. We have shown that the degeneracy exists for an arbitrary number of impurities contributing to pinning the bisoliton. Experimentally there exists also slow relaxation in commensurate systems, even though faster than in the incommensurate case. The fully classical model might be too schematic since the exact degeneracy of the classical model is lifted by quantum tunneling. Nevertheless it succeeds to explain the $C_{v} \sim T^{-2}$ specific heat and the waiting time dependence of the prefactor in the incommensurate case. We have proposed a model of clustering in which the pinning energy is additive if two impurities are at a distance smaller than the width $\xi$ of the soliton. The barrier distribution is exponential, with therefore a powerlaw relaxation compatible with experiments (see Ref.12). However bisolitons can be depinned in sequence, not simultaneously. This is described qualitatively by the dynamical renormalization group 12 . Within this treatment we obtain also a power-law relaxation but with an upper cut-off in the spectrum of relaxation times, in agreement with experiments. The clustering model is nevertheless 
useful for addressing qualitatively the waiting time effect in the specific ageing protocol used in experiments.

Finally we discussed another important factor: the nature of disorder at the level of a single soliton and the possibility of quantum interactions among solitons that might explain the differences between the spin-Peierls compound $\mathrm{Cu}_{1-x} \mathrm{Zn}_{x} \mathrm{GeO}_{3}$ that does not show slow relaxation and orders antiferromagnetically, and the spinPeierls compound (TMTTF) ${ }_{2} \mathrm{PF}_{6}$ that shows slow relaxation without antiferromagnetic order. For this purpose we have generalized to incommensurate systems a model of substitutional disorder originally introduced for spinPeierls systems 34 . We first treated the case of a strong pinning impurity potential and found that the energy spectrum of a single quantum mechanical soliton is constant as the position of the soliton varies along the chain in the commensurate case, whereas it varies in the incommensurate case. Interactions among solitons enhance disorder effects in the incommensurate case. We thus find a qualitative difference between the commensurate and incommensurate cases in the quantum limit. For substitutional disorder in the quantum limit we find interactions among solitons due to Friedel oscillations. This can explain the experimentally observed power-law contribution to the specific heat in inorganic CDWs and SDWs in the limit of weak interchain couplings.

The final picture for organic SDWs and CDWs is a coexistence between strong pinning and substitutional disorder as well as a a coexistence between classical and quantum effects. Further investigations would require a model of collective effects interpolating between the classical and quantum limits. Another ingredient that we did not discuss in detail is metallic island formed around impurities 43 that would lead to a phenomenology close to that of substitutional disorder because both generate states at the Fermi level for an isolated impurity, that can interact through Friedel oscillations.

Finally, we have developed here the point of view of including collective effects from the strong pinning limit. The specific heat including quantum effects in the weak pinning regime was discussed recently 44 .

\section{Acknowledgments}

The authors acknowledge fruitful discussions with $\mathrm{P}$. Monceau. K.B. acknowledges funding by CNRS through a temporary position of associate researcher. The authors thank one of the referees for useful comments on the first version of the manuscript.

\section{APPENDIX A: GREEN'S FUNCTIONS OF A CHARGE DENSITY WAVE}

\section{Canonical transformation}

We use the Peierls Hamiltonian for charge degrees of freedom, the electronic part of which is given by Eq. (29), where spinless fermions jump between neighboring sites on a $1 \mathrm{D}$ chain. We suppose that the lattice is frozen and therefore we do not include in the Hamiltonian the term due to the lattice deformations. The Hamiltonian (29) is diagonal in terms of the operators $\gamma_{k, R}$ and $\gamma_{k, L}$, where $\mathrm{R}$ and $\mathrm{L}$ label right and left-moving fermions:

$$
\mathcal{H}=\sum_{k}\left[E_{k, R} \gamma_{k, R}^{+} \gamma_{k, R}+E_{k, L} \gamma_{k, L}^{+} \gamma_{k, L}\right],
$$

with

$$
\begin{aligned}
\gamma_{k, R}^{+} & =\mathcal{N}_{k}^{(R)}\left[c_{k, R}^{+}+\mathcal{B}_{k}^{(R)} c_{k-2 k_{F}, L}^{+}\right] \\
\gamma_{k, L}^{+} & =\mathcal{N}_{k}^{(L)}\left[c_{k, L}^{+}+\mathcal{B}_{k}^{(L)} c_{k+2 k_{F}, R}^{+}\right]
\end{aligned}
$$

and

$$
\begin{aligned}
& E_{k, R}=2 t \cos \left(k a_{0}\right)-\frac{\epsilon^{2}}{4 t \sin \left(k_{F} a_{0}\right)} \frac{1}{\left(k-k_{F}\right) a_{0}}(\mathrm{~A} 4) \\
& E_{k, L}=2 t \cos \left(k a_{0}\right)+\frac{\epsilon^{2}}{4 t \sin \left(k_{F} a_{0}\right)} \frac{1}{\left(k+k_{F}\right) a_{0}}(\mathrm{~A} 5)
\end{aligned}
$$

The coefficients $\mathcal{B}_{k}^{(R)}$ and $\mathcal{B}_{k}^{(L)}$ are given by

$$
\begin{aligned}
\mathcal{B}_{k}^{(R)} & =-\frac{\epsilon}{4 t} \frac{\exp \left(i k_{F} a_{0}\right)}{\left(k-k_{F}\right) a_{0}} \\
\mathcal{B}_{k}^{(L)} & =\frac{\epsilon}{4 t} \frac{\exp \left(-i k_{F} a_{0}\right)}{\left(k+k_{F}\right) a_{0}}
\end{aligned}
$$

The normalization coefficients are given by $\mathcal{N}_{k}^{(R, L)}=$ $1 / \sqrt{1+\left|\mathcal{B}_{k}^{(R, L)}\right|^{2}}$. The Green's functions deduced from the spectral representations are given in Appendix $\mathrm{A}$

\section{Green's functions}

The advanced Green's function defined as $g_{y_{1}, y_{2}}\left(t_{1}, t_{2}\right)=-i \theta\left(t_{1}-t_{2}\right)\left\langle\left\{c_{y_{1}}\left(t_{1}\right), c_{y_{2}}\left(t_{2}\right)\right\}\right\rangle$ decomposes into the sum of the four right $(\mathrm{R})$ and left $(\mathrm{L})$ combinations:

$$
\begin{aligned}
g_{y_{1}, y_{2}}\left(t_{1}, t_{2}\right) & =g_{y_{1}, y_{2}}^{R, R}\left(t_{1}, t_{2}\right)+g_{y_{1}, y_{2}}^{R, L}\left(t_{1}, t_{2}\right) \\
& +g_{y_{1}, y_{2}}^{L, R}\left(t_{1}, t_{2}\right)+g_{y_{1}, y_{2}}^{L, L}\left(t_{1}, t_{2}\right),
\end{aligned}
$$

where the "RR" Green's function is defined by

$$
g_{y_{1}, y_{2}}^{R, R}\left(t_{1}, t_{2}\right)=\sum_{k_{1}, k_{2}} e^{i k_{1} y_{1}} e^{-i k_{2} y_{2}}\left\langle\left\{c_{k_{1}, R}\left(t_{1}\right), c_{k_{2}, R}\left(t_{2}\right)\right\}\right\rangle,
$$


and similar expressions are obtain for the "RL", "LR" and "LL" Green's functions. The spectral representation of $g_{y_{1}, y_{2}}^{R, R}$ is given by

$$
\begin{aligned}
g_{y_{1}, y_{2}}^{R, R}(E) & =\sum_{k}\left[\mathcal{N}_{k}^{(R)}\right]^{2} e^{i k\left(y_{1}-y_{2}\right)} \\
& \times\left\{\frac{1}{E-E_{k, R}}+\frac{\left|\mathcal{B}_{k}^{(R)}\right|^{2}}{E-E_{k-2 k_{F}, L}}\right\},
\end{aligned}
$$

and similar expressions are obtained for the three other Green's functions. After performing the integral over wave vector in the spectral representations we obtain

$$
\begin{aligned}
& g_{y_{1}, y_{2}}^{R, R}(E)+g_{y_{1}, y_{2}}^{L, L}(E)=\frac{2}{3} \frac{1}{2 t \sin k_{F}} \frac{\sqrt{2} \epsilon}{\sqrt{2 \epsilon^{2}-E^{2}}} \\
& \sin \left\{\varphi+\left[k_{F}-\frac{E}{4 t \sin k_{F}}\right] R\right\} \exp (-R / \xi(E)) \\
+ & \frac{1}{3} \frac{1}{2 t \sin k_{F}} \frac{\sqrt{2} \epsilon}{\sqrt{2 \epsilon^{2}-E^{2}}}
\end{aligned}
$$

1 L. Lundgren, P. Svedlindh, P. Nordblad and O. Beckmann, Phys. Rev. Lett. 51, 911 (1983); P. Nordblad, L. Lundgren, P. Svedlindh and L. Sandlund, Phys. Rev. B 33, 645 (1988).

2 V. Dupuis, E. Vincent, J.P. Bouchaud, J. Hammann, A. Ito, and H. Aruga Katori, cond-mat/0104399 K. Jonason, E. Vincent, J. Hammann, J.P. Bouchaud, and P. Nordblad, Phys. Rev. Lett. 81, 3243 (1998); K. Jonason, P. Nordblad, E. Vincent, J. Hammann, and J.P. Bouchaud, Eur. Phys. Jour. B 13, 99 (2000).

3 J. Gilchrist, Phys. Lett. A 156, 76 (1989), J. of Mol. Liq. 69, 253 (1996).

${ }^{4}$ F. Alberici, P. Doussineau and A. Levelut, J. Phys. I (France) 7, 329 (1997); F. Alberici, P. Doussineau and A. Levelut, Europhys. Lett. 39, 329 (1997).

${ }^{5}$ F. Alberici-Kious, J.P. Bouchaud, L.F. Cugliandolo, P. Doussineau and A. Levelut, Phys. Rev. Lett. 81, 4987 (1998); F. Alberici-Kious, J.P. Bouchaud, L. F. Cugliandolo, P. Doussineau and A. Levelut, Phys. Rev. B 62, 14766 (2000).

${ }^{6}$ R.L. Leheny and S. Nagel, Phys. Rev. B 57, 5154 (1998).

7 J.C. Lasjaunias, K. Biljaković, D. Staresinic, P. Monceau, S. Takasaki, J. Yamada, S. Nakatsuji, and H. Anzai, Eur. Phys. J. B 7, 541 (1999).

8 K. Biljaković, J.C. Lasjaunias, P. Monceau and F. Levy, Phys. Rev. Lett. 62, 1512 (1989); K. Biljaković, J.C. Lasjaunias, P. Monceau and F. Levy, Phys. Rev. Lett. 67, 1902 (1991).

9 J.C. Lasjaunias, J.P. Brison, P. Monceau, D. Staresinic, K. Biljaković, C. Carcel and J. M. Fabre, J. Phys.: Condens. Matter 14837 (2002).

${ }^{10}$ K. Biljaković, F. Nad, J.C. Lasjaunias, P. Monceau, and K. Bechgaard, J. Phys. Condens. Matter 6, L135 (1994); K. Biljaković, F. Nad, J.C. Lasjaunias, P. Monceau, and K. Bechgaard, Synthetic Metals 71, 1849 (1995); J.C. Lasjaunias, K. Biljaković, and P. Monceau, Phys. Rev. B 53, 7699 (1996); J.C. Lasjaunias, S. Sahling, K. Biljaković, and

$$
\sin \left\{\varphi-\left[k_{F}+\frac{E}{4 t \sin \left(k_{F} a_{0}\right)}\right] R\right\} \exp (-R / \xi(E))
$$

where $R=y_{1}-y_{2}$ is positive and $\xi(E)=$ $4 t \sin \left(k_{F} a_{0}\right) / \sqrt{2 \epsilon^{2}-E^{2}}$ is the coherence length at a finite frequency. The sum of the "RL" and "LR" Green's functions is given by

$$
\begin{aligned}
& g_{y_{1}, y_{2}}^{R, L}(E)+g_{y_{1}, y_{2}}^{L, R}(E)= \\
- & \frac{\sqrt{2}}{3} \frac{1}{2 t \sin \left(k_{F} a_{0}\right)} \frac{\sqrt{2} \epsilon}{\sqrt{2 \epsilon^{2}-E^{2}}} \exp (-R / \xi(E)) \\
& \left\{\cos \left[k_{F}+2 \varphi+\left[k_{F}-\frac{E}{4 t \sin \left(k_{F} a_{0}\right)}\right] R-2 k_{F} y_{1}\right]\right. \\
- & \left.\cos \left[k_{F}-\left[k_{F}-\frac{E}{4 t \sin \left(k_{F} a_{0}\right)}\right] R-2 k_{F} y\right]\right\} .
\end{aligned}
$$

We obtain similar expressions for $R<0$.
P. Monceau, J. of low T. Phys. 130, 25 (2003).

11 J.P. Bouchaud, J. Phys. I France 2, 1705 (1992); J.P. Bouchaud, E. Vincent, and J. Hamman, J. Phys. I France 4, 139 (1994); J.P. Bouchaud and D.S. Dean, J. Phys. I France 5, 265 (1995). C. Monthus and J.P. Bouchaud, J. Phys. A 29, 3847 (1996).

12 R. Mélin, K. Biljaković, J.C. Lasjaunias and P. Monceau, Eur. Phys. J. B 26, 417 (2002).

13 S.K. Ma, C. Dasgupta and C.-K. Hu, Phys. Rev. Lett. 43, 1434 (1979); C. Dasgupta and S.K. Ma, Phys. Rev. B22, 1305 (1980).

14 D.S. Fisher, P. Le Doussal and C. Monthus, Phys. Rev. Lett. 80, 3539 (1998); D.S. Fisher, P. Le Doussal and C. Monthus, Phys. Rev. E 64, 066107 (2001).

15 H. Fukuyama, J. Phys. Soc. Jpn. 41, 513 (1976).

16 H. Fukuyama and P. A. Lee, Phys. Rev. B 17, 535 (1977); H. Fukuyama, J. Phys. Soc. Jpn. 45, 1474 (1978); P. A. Lee and T. M. Rice, Phys. Rev. B 19, 3970 (1979).

17 K. Efetov and A.I. Larkin, Zh. Eksp. Teor. Fiz. 72, 2350 (1977) [Sov. Phys. JETP 45, 1236 (1977)].

18 A.I. Larkin and P. Lee, Phys. Rev. B 17, 1596 (1978).

19 S. Abe, Physica 143 B, 85 (1986).

20 P.B. Littlewood and R. Rammal, Phys. Rev. B 38, 2675 (1988).

21 S. Brazovskii, Charge Density waves in solids, edited by L. Gorkov and G. Grüner (Elsevier, Amsterdam) (1990).

22 A.I. Larkin, Zh. Eksp. Teor. Fiz. 105, 1793 (1994); A.I. Larkin Sov. Fiz. JETP 78, 971 (1994).

${ }^{23}$ Yu. N. Ovchinikov, K. Biljakovic, J.C. Lasjaunias, and P. Monceau, Europhysics Letters 34, 645 (1996).

24 S. Brazovskii and T. Nattermann, Advances in Physics 53, 177 (2004).

25 B. Derrida, Phys. Rev. B 24, 2613 (1981); B. Derrida and G. Toulouse, J. Phys. Lett. 46, L223 (1985).

26 S. Ravy, J.P. Pouget and R. Comes, J. Phys. I France 2, 1173 (1992); S. Rouzière, S. Ravy, J.P. Pouget and S. Brazovskii, Phys. Rev. B 62, R16232 (2000); J.P. Pouget, 
S. Ravy, S. Rouzière and S. Brazovskii, J. Phys. IV France 12, Pr9-9 (2002); S. Ravy, S. Rouzière, E. Elkaïm, J.P. Pouget and S. Brazovskii, ibid 12, Pr9-79 (2002).

27 K. Biljakovic, M. Miljak, D. Staresinic, J. C. Lasjaunias, P. Monceau, H. Berger and F. Levy, Eurphys. Lett. 62, 554 (2003).

28 J. Dumas, J.C. Lasjaunias, K. Biljakovic, M. Miljak, H. Berger, F. Levy, Solid State Commun. 132, 661 (2004).

29 J.C. Lasjaunias, P. Monceau, D. Staresinic, K. Biljakovic, C. Carcel, J.M. Fabre, J. Phys. Cond. Matter 14, 8583 (2002).

30 M. Hase, K. Uchinokura, R.J. Birgeneau, K. Hirota and G. Shirane, J. Phys. Soc. Jpn. 65, 1392 (1996);

M. Hase, N. Koide, K. Manabe, Y. Sasago, K. Uchinokura and A. Sawa, Physica B 215, 164 (1995).

31 M.C. Martin, M. Hase, K. Hirota and G. Shirane, Phys. Rev. B 56, 3173 (1997).

32 T. Masuda, A. Fujioka, Y. Uchiyama, I. Tsukada, and K. Uchinokura, Phys. Rev. Lett. 80, 4566 (1998).

${ }^{33}$ K. Manabe, H. Ishimoto, N. Koide, Y. Sasago, and K. Uchinokura, Phys. Rev. B 58, R575 (1998).

34 M. Fabrizio and R. Mélin, Phys. Rev. Lett. 78, 3382 (1997); M. Fabrizio and R. Mélin, Phys. Rev. B 56, 5996 (1997); M. Fabrizio. R. Mélin and J. Souletie, Eur. Phys. J. B 10, 607 (1999); R. Mélin, Eur. Phys. J. B 16, 261 (2000); R. Mélin, Eur. Phys. J. B 18, 263 (2000).
35 D. Staresinic, K. Hosseini, W. Bruetting, K. Biljakovic, E. Riedel, S. van Smaalen, Phys. Rev. B 69, 113102 (2004).

36 J.C. Lasjaunias, K. Biljakovic, F. Nad, P. Monceau, and K. Bechgaard, Phys. Rev. Lett. 72, 1283 (1994).

37 G. Grüner, Density waves in Solids, Frontiers in Physics, vol. 89 (1994).

38 T. Takoshima, M. Ido, K. Tsutsumi, T. Sambongi, S. Houma, K. Yamaya, abd Y. Abs, Solid State Commun. 35, 911 (1980).

39 R.J. Glauber, Jour. Math. Phys. 4, 294 (1963).

40 F. Nad, P.Monceau and K. Bechgaard, Solid State Commun. 95, 655 (1995), and refences therein.

41 M. Laukamp, G.B. Martins, C. Gazza, A.L. Malvezzi, E. Dagotto, P.M. Hansen, A.C. Lopez and J. Riera, Phys. Rev. B 57, 10755 (1998).

42 D. Augier, E. Sørensen, J. Riera, and D. Poilblanc Phys. Rev. B 60, 1075 (1999); A. Dobry, P. Hansen, J. Riera, D. Augier, and D. Poilblanc, Phys. Rev. B 60, 4065 (1999); E. Sørensen, I. Affleck, D. Augier, and D. Poilblanc, Phys. Rev. B 58, R14701 (1998).

43 S.N. Artemenko and F. Gleisberg, Phys. Rev. Lett. 75, 497 (1995).

44 G. Schehr, T. Giamarchi, and P. Le Doussal, Europhys. Lett. 66, 538 (2004). 